\title{
ATTITUDES TOWARDS THE USE OF MOBILE HEALTH APPLICATIONS IN BULGARIA
}

\section{Kristina Kilova $^{1}$, Desislava Bakova ${ }^{2}$, Antonia Yaneva ${ }^{3}$, Vanina Mihaylova ${ }^{4}$}

\begin{abstract}
Background: The increasing use of mobile phones has offered an opportunity to improve the quality of health services, secure the accessibility of healthcare systems for a larger population and support patients in effective management of their health/chronic conditions.

Main Goal: The primary focus of the study in hand is to explore the different attitudes towards the use of mHealth in Bulgaria and to examine the extensive use of mobile health applications for the supervision, prevention and containment of various socially relevant diseases, including the promotion of healthy lifestyles.

Materials and methods: An online survey was conducted in the period between July 2019 and February 2020. Amongst the 976 people that were interviewed, $591(60.6 \%)$ were females and $385(39.4 \%)$ were males. The mean age of the respondents was $43.25 \pm 0.755$. A unique questionnaire was prepared for the collection of information regarding the usage of mobile health applications. Statistical analyses were carried out with the help of IBM SPSS Statistics, version 23. MS Excel 2016 was used for the graphical representation of the results.

Results: $89 \%$ of the participants possessed a mobile phone and an approximate $84 \%$ of them used mHealth applications. Besides this, the results suggested a considerable statistical difference in the respondents' attitudes, in terms of their gender, where the deviation was in favour of female respondents. Additionally, findings revealed another statistical difference on the basis of their age and level of education. Furthermore, a huge majority claimed that they generally used these types of applications for monitoring their blood pressure or pulse.

Conclusion: The outcome of the study was that a significant number of respondents were already using or tended to use smartphones and various applications, including health ones. We expect that the results of this study will provide evidence of the essentiality of mobile health applications to be developed in Bulgaria. This is crucial for patients as this technology has the potential to open up several new possibilities for treatments and observations of diverse socially significant diseases.
\end{abstract}

UDC Classification: 606:628, DOI: https://doi.org/10.12955/pmp.v1.98

Keywords: mHealth, mobile health applications, telemedicine.

\section{Introduction}

Mobile Health (mHealth) is recognised as the most convenient way for remote health service delivery and real-time data collection, thus improving disease control and treatment by way of delivering care from the hospital to the confines of someone's home. It offers better accessibility to healthcare systems, a development in the patients' understanding or knowledge of their own health/medical conditions and an improvement in their promptitude to engage in self-management, allowing high-quality care and satisfying both the patients and healthcare professionals (Chhabra et al., 2018).

In 2016, the World Health Organisation (WHO) Executive Board defined Mobile health (mHealth) as: "... the use of wireless mobile technologies for public health..." demonstrating the increasing relevance of the resource for the provision of quality health services and public health, while keeping in mind its convenience, prevalence and wide approbation. As testified, mHealth improves access to information on health, to the services, assistance and skills of professionals or specialists, and also directs positive change in health behaviour and disease management (WHO, 2016).

As mobile health is mainly performed through mobile phones nowadays, they provide the opportunity to ensure accessibility of health services to a larger number of individuals and to strengthen the capacity of the healthcare system. Various mobile applications measure blood pressure, remind us of our drug intake, send insulin reports to diabetics, improve mental health, etc. (Vinarova, 2015)

Global mobile subscriptions rose by $1.5 \%$, quarter-on-quarter, and around 5\%, year-on-year (Ericson, 2015). As of January 2019, approximately two-thirds of the world population (or 5.11 billion out of

\footnotetext{
${ }^{1}$ Medical University of Plovdiv, Bulgaria: Dept. "Medical Informatics, Biostatistics and E-Learning”, Faculty of Public Health, k_kilova@yahoo.com

${ }^{2}$ Medical University of Plovdiv, Bulgaria: Dept. "Health Care Management", Faculty of Public Health, desislavabakova@abv.bg

${ }^{3}$ Medical University of Plovdiv, Bulgaria: Dept. "Medical Informatics, Biostatistics and E-Learning”, Faculty of Public Health, antoniya.yaneva@mu-plovdiv.bg

${ }^{4}$ Medical University of Sofia, Bulgaria: Dept. "Physiotherapy", Faculty of Public Health; Medical University of Plovdiv, Bulgaria: Bulgaria: Dept. "Health Care Management”, Faculty of Public Health, vanina_delfi@abv.bg
} 
7.676 billion) possessed mobile devices and three-fifths (4.39 billion) were Internet users. Social network users also accounted for three-fifths of the world's population as their numbers internationally increased to 288 million (9\%), eversince January 2018 (Kemp, 2019). This data illustrates the significance of mobile phones in the Internet space today. The widespread use of mobile phones also relates to the innovation of different applications, one of which is Mobile Health.

The term 'Mobile Health' (mHealth) is used for the practice of medicine and public health supported by gadgets (Asangansi \& Braa, 2010), such as mobile phones, patient monitoring devices, personal digital assistants and wireless devices. The spheres of its application are manifold:

- For Communication Between the Patient and Health Professionals - it provides help-lines, free calls in case of emergencies, reminder messages or alarms for drug intake or examination appointments, bookings for consultations from medical specialists, cross-sector communication in emergencies and various disasters.

- Patient Monitoring - it helps in the collection and transfer of observed data and in the recording of diverse conditions in different settings using mobile information and communication technology (ICT).

- It helps in data compiling and management and in submitting health tests with the use of mobile ICT.

- Access to Information and Training of Healthcare Specialists - it offers mobile learning (mLearning) and access to online educational resources through mobile ICT.

- Access to Electronic Patient Information - one can retrieve laboratory results, radiographs, etc. using mobile ICT (WHO, 2011).

Mobile Health is an inexpensive and simple means of exchanging health information, which is why it is being advocated by the UN, governments, non-governmental organizations and academic institutions (Hampton, 2012).

In 2018, the 71st World Health Assembly (WHA) encouraged its member countries to adopt several policies in order to introduce digital technologies in healthcare systems (WHO, 2018). As a result of the assembly, a resolution called the Resolution WHA71.7 was passed, which recognized the potential of digital technologies in enhancing public health.

Resolution EB139/8 (2016) acknowledged mHealth as a means to ameliorate accessibility of health information and services as well as advance health promotion. With Resolution EB142/20 (2018), WHO approved this updated report on mHealth.

A substantial advantage that mobile technologies provide is that they have a peculiar manner of assembling health information and the ability to supply personalized care. The use of mobile healthcare also presents an opportunity to reduce health costs by means of early prevention, early disease diagnosis and by saving work time for healthcare workers.

Mobile technology is rapidly transforming health care, education, and research (Marcolino et al., 2018; Mohapatra et al., 2015). The growing availability of smartphones has allowed the rapid development and use of smartphone applications. Mobile health interventions formerly relied on voice or text-based short message services (SMS), but now there are many applications which can be used for changes in health behaviour (McKay et al., 2018).

The number of smartphone applications aimed at improving health is ever-increasing. According to a survey by the Pew Research Center, $19 \%$ of mobile phones owners use such applications. A few of the latest mobile phone applications in respect to nutrition and calorie intake are DietPoint, Fooducate, Lose it, Lifesum, etc. Other applications such as 'FitBit' contribute in the physical activity of consumers (Fox \& Duggan, 2012). 'Calm' is a mental health application which provides mental exercises and relaxation and meditation techniques. 'Sleep Cycle' assesses the patient's sleep. There is a lot of other applications such as 'iOS Health' and 'CareZone', which provide an integration of the overall health information of an individual.

The application, CAIDE (Cardiovascular Risk Factors, Aging, and Incidence of Dementia), is worth mentioning as it is an evidence-based instrument which predicts the chances of developing late dementia on the basis of age, educational level and other risk-contributing factors (like hypertension, hypercholesterolemia, obesity, physical inactivity, etc.) (Sindi et al., 2015). This application and the 
ones akin to it, provide users with prospects to evaluate their health condition and, if suitable, to undertake preventive measures against the issue.

Other innovative ICT applications that have a broader scope and concern human health are Apple Watch, AliveCor, ZEUS Robotic Surgical System, Da Vinci Surgical System etc. (Choi et al., 2018).

Mobile health applications such as those which help in self-managing diabetes prove to be really useful in improving the control of glycated haemoglobin levels by providing a feedback loop between the patient and the healthcare provider. Similar applications incorporate behaviour-based interventions for enhancing patient access to diabetes self-management education and facilitating effective support. Most of the studies related to the mHealth applications were executed under controlled conditions, but they did not analyse the perceptions of patients and the efficacy of these applications in everyday life (Debong et al., 2019).

As a member state of the European Union, Bulgaria follows the European policy and strategy for achieving smart, sustainable, and inclusive digital growth for the time period up to 2025. In 2019, Bulgaria adopted a national program 'Digital Bulgaria 2025', which intended to upgrade and enforce information technology solutions in every sector of the economy and social life (Decision №730/05-122019, Ministry of Transport, Information Technology and Communications). According to the latest TEMPEST mHealth report (Currie \& Seddon, 2014), which uses integrative approach to understand the complexities and challenges of introducing healthcare technologies and juxtaposes data from $28 \mathrm{EU}$ members, Bulgaria ranks sixth with the highest percentage in information technology investment, but with the lowest percentage of households with Internet access. Simultaneously, between the years 2005 and 2012, the mobile traffic in Bulgaria increased by over 200\%. The Eurostat data reveals that in 2019, the fraction of people in the age group 16-74 years, who claimed that they sought information online for health-related topics, was highest in Finland, where 76\% of Internet users searched online for health information. The lowest proportions were observed in Bulgaria (30\%), Romania (31\%) and Italy (35\%) (Eurostat, 2020).

The coronavirus epidemic (COVID-19) poses a novel challenge for public health around the world (Anderson et al, 2020). Oliver et al. (2020) gave suggestions on how mobile phone data can counterwork the virus and explained the ways in which it can aid in controlling and probably even slowing down the spread of the virus. In the course of the current crisis, the Bulgarian Ministry of Health launched a national information system for combating COVID-19. It provides information on all people diagnosed and quarantined and links all relevant institutions. Additionally, a free mobile app, ViruSafe, which facilitates health status sharing with the national operational staff, health authorities and general practitioners, was developed.

\section{Materials and Methods}

An online survey was conducted in the period of July 2019 to February 2020, in which 976 people were interviewed. An innovative questionnaire was designed containing 18 questions divided into two panels. The first panel covered questions related to demographic characteristics of the participants (gender, age, education, place of residence). The second panel consisted of questions related to mHealth attitudes and widespread usage of mobile health applications, which were measured on a 5-point Likert scale. The scale ranged from "complete disagreement" to "complete agreement". The indices with the values 1 or 2 corresponded to dissatisfaction; the index value 3 meant neutral satisfaction; and the indices 4 or 5 indicated complete satisfaction of the respondents. Five of the questions had only one answer, whereas two of the questions had more than one answer.

Descriptive statistics was used to review the frequency, mean and standard deviation for the demographic characteristics as well as for the variables related to the use of mobile phones and mobile applications. The study employed a quantitative cross-sectional descriptive framework to provide an outline and assessment of the prevalence and usage of mobile health (mHealth) applications in Bulgaria. Criterion ' $\chi$ ' was used for the comparison of the results in two-dimensional distributions and the level of significance for the null hypothesis was ' $\mathrm{P}<0.05$ '. All statistical analyses were conducted using SPSS, version 23. MS Excel 2016 was used for graphical representation of the results.

\section{Results}

In our study 976 people ( $\geq 18$ years old) were surveyed, in which $591(60.6 \%)$ were females and 385 (39.4\%) were males. The participants were segregated into four age groups - 18-24 years (13\%), 25-49 
(50\%), 50-64 (31\%), and over 65 years of age (6\%). The end results showed that $50 \%$ of respondents came under the active working age group (25-49 years), and that less than $6 \%$ of people were in the retirement age group. This distribution in terms of age groups is presented in figure 1.

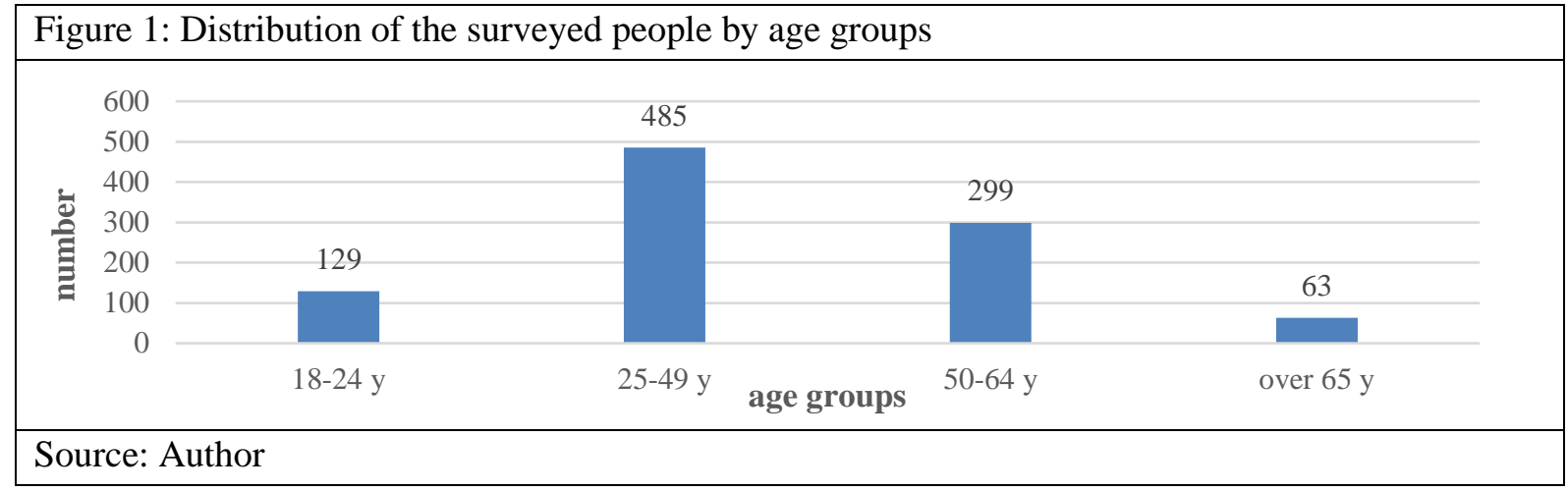

The mean age of the participants was $43.25 \pm 0.755$ years (ranging between $18-79$ years).

The sample was also divided on the basis of educational level, in which $0.7 \%(n=7)$ respondents had completed primary education and more than $75 \%$ of them had a bachelor`s, master`s or $\mathrm{PhD}$ degree. This data according to educational level is presented in figure 2.

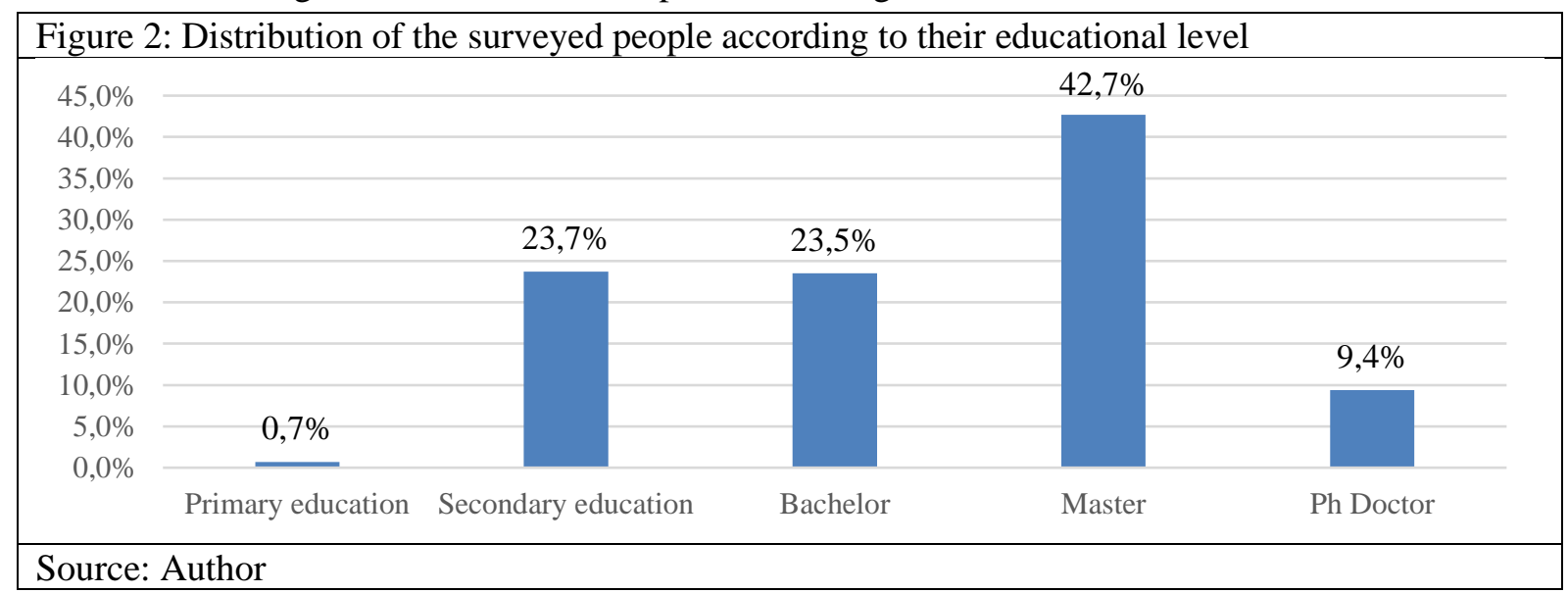

More than $82 \%(n=801)$ of the interviewees had daily access to the Internet, about $89 \%(n=869)$ had smartphones, and approximately $83.5 \%$ used mobile applications on their phones for support, assistance or management of their chronic diseases.

To the question: "How many health-related applications do you have on your mobile phone?", 58.9\% $(n=575)$ of the respondents declared that they used 1 to 2 mHealth applications. The frequency distribution of the answers is presented in figure 3.

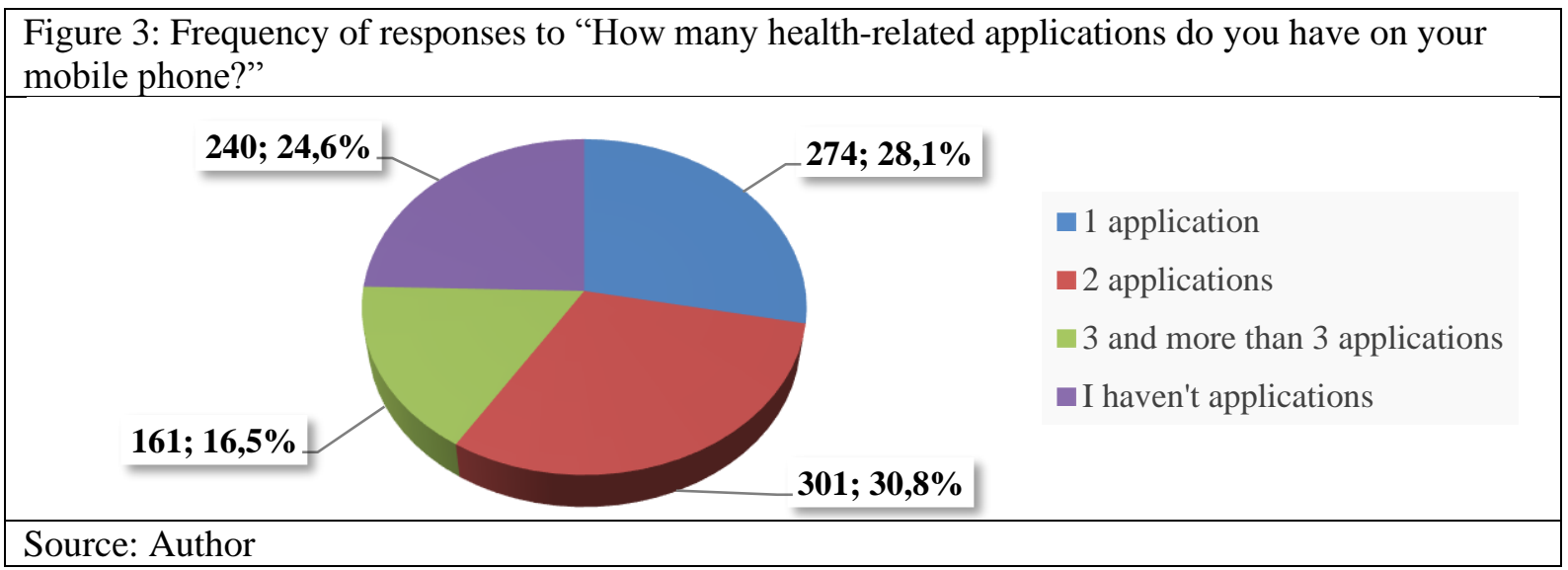

Over $68 \%$ of the sample population used their mobile phones to access information regarding their own health. A statistically significant difference was found between the four age groups and their usage of 
mobile applications $\left(\chi^{2}=48.221 ; \mathrm{P}<0.01\right)$. The younger respondents were more likely to use different mobile applications than older ones, but more than $63 \%$ of all participants expressed an interest in using their mobile phones to monitor their disease progression.

To the question: "What type of mobile application do you use or would you use to monitor your health?", a large majority of subjects $(89.4 \%, \mathrm{n}=869)$ responded that they use mHealth applications mainly for keeping a check on their blood pressure and pulse and for the self-management of blood sugar levels $(69.4 \%, \mathrm{n}=677)$. Another section of participants (over 60\%), claimed that weight loss apps help them keep track of their food habits and promote healthy lifestyles $(61.9 \%, n=604)$. The proportion of the sample that used mobile applications for diagnoses and medication was 60.8\% $(n=593)$ (Figure 4).

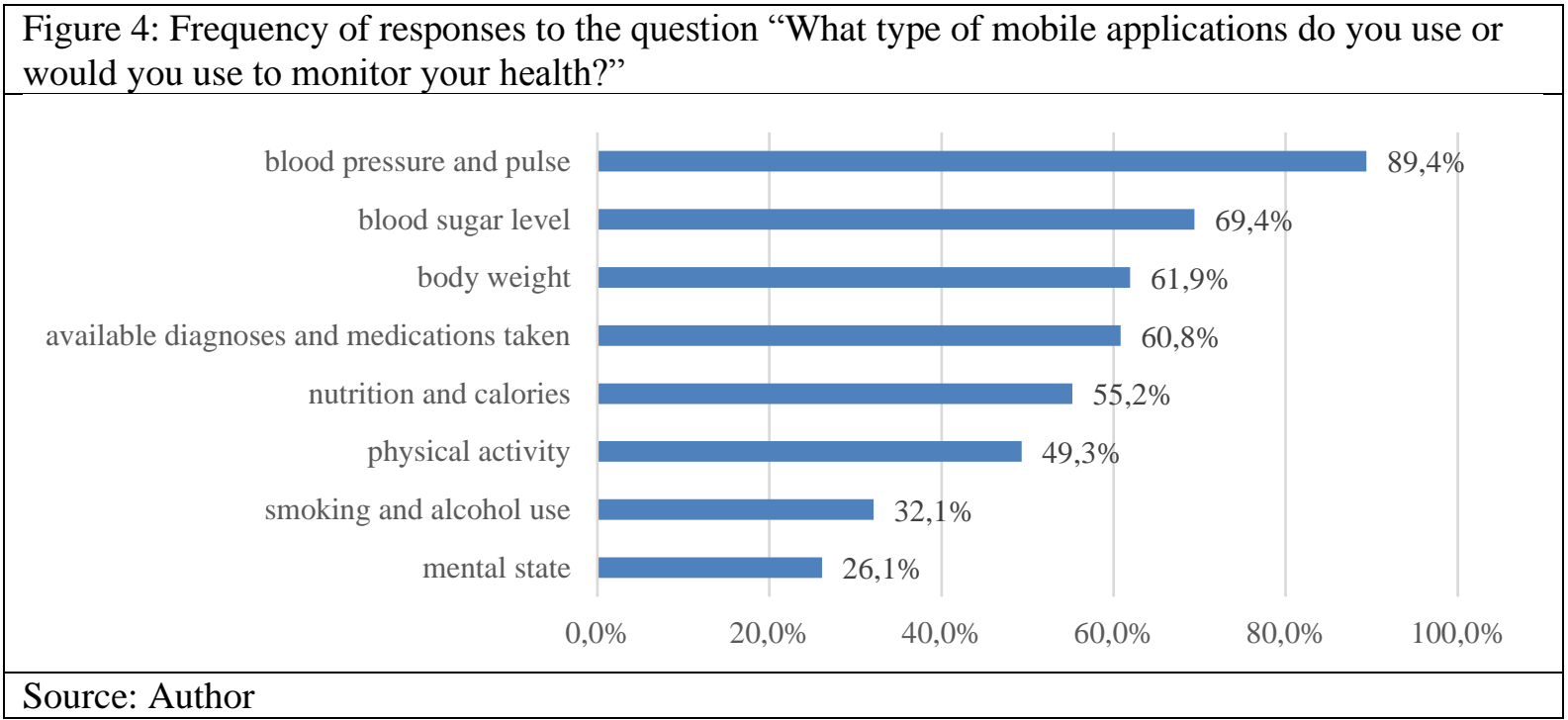

Above $50 \%$ of the respondents used or desired to use mobile applications to record and oversee their blood pressure, heart rate, body weight, blood sugar, the appropriate diagnoses and medications, and the nutrition and calories consumed. $49.3 \%$ of them were also interested in physical activity applications.

While scrutinizing the disparate attitudes of respondents towards the subscription and exchange of personal health data by means of a mobile device or application, resultant replies revealed that they encountered issues whilst working with mobile technology $(57 \%)$ and that they were afraid of misinterpretation of information (58\%). Likewise, the fact that $42 \%$ of the respondents have concerns pertaining to the discretion of their personal health information and the possibility of it falling into the wrong hands, is a point which needs emphasis. A huge majority of respondents voice their concerns on the possibility of their personal information being misused, with only $35 \%$ of them stating that they do not see any risk. A statistically significant difference was found between the preparedness to monitor one's health with the help of a mobile application and the sharing of personal information $\left(\chi^{2}=37.665\right.$; $\mathrm{P}<0.001)$. There were statistically significant differences in the answers of the respondents on the basis of their gender $\left(\chi^{2}=37.825 ; \mathrm{P}<0.05\right)$ as well. Women were more likely to subscribe to mobile health applications in comparison to men.

Another statistically significant difference occurred in the answers of the respondents owing to their age and level of education. Older respondents $\left(\chi^{2}=356.843 ; \mathrm{P}<0.05\right)$ and less educated people $\left(\chi^{2}=132.362\right.$; $\mathrm{P}<0.05)$ are unlikely to subscribe to mobile applications and share health related personal data as compared to younger ones.

Around $64 \%$ of participants agreed to use a mobile application in order to monitor or control their health. On the probability that the use of health applications would actually impact their health, $62.9 \%$ of respondents reported that they do not expect any improvements in their health.

\section{Discussion}

Mobile health applications are rapidly being used for the purpose of substituting the conventionally performed tasks of general practitioners, such as those involved in promoting health, preventing diseases, making diagnoses, giving treatments, monitoring health status, and supporting or assisting in health services (Wattanapisit et al., 2020). 
In this research, we investigate the attitudes towards mHealth applications and the use of smart devices for health promotion and for the support and management of health statuses among Bulgarian population. As a summary, the analyses indicated that mobile health application users were generally young and well educated and that women were more likely to use such applications. The most common type of health applications mentioned were those that enabled monitoring of diseases, like hypertension, diabetes, obesity, etc. These findings are vital because they are of social significance in Bulgaria.

The results of a recently held study on the attitudes towards the use of mobile applications for blood pressure indicate that $89.4 \%$ people support those found through a survey among workers and students in South Korea (94.3\%). However, their blood sugar results (93.6\%) differ from ours (69.4\%) (Jeon \& Seo, 2016). Our findings on the use of a mobile blood sugar application, back those of the study by Muigg D et al. (2018) on the willingness of patients to use telemonitoring systems for diabetes in Austria (65\%). The application for blood sugar is especially advanced. For example, a meta-analysis involving 42 randomized controlled trials of $\mathrm{HbA} 1 \mathrm{c}$ changed after routine care and telemedicine intervention found that the reduction in $\mathrm{HbA} 1 \mathrm{c}$ was contrastingly greater in telemedicine groups, particularly when telemedicine programs lasted more than 6 months as this is more applicable to patients with type 2 diabetes (Tchero et al, 2018). The use of ICT is an opportunity for the prevention, early diagnosis, registration and more effectual treatment of hypertension, diabetes and obesity.

The fact that only $26.1 \%$ of respondents would use a mobile application for their mental state reflects their reluctance and hesitation to share personal socio-psychological problems. Nevertheless, in Saudi Arabia, an online survey among the mentally ill found that $64 \%$ of them used their mobile phones to keep a check on their depression and/or anxiety (Atallah et al., 2018). Global studies on mental health applications show interesting results. For example, a study in Poland on the impact of a smartphone application in the field of psychiatry, which enabled remote contact with a doctor and adjustments in the dosage of the treatment, found that after one year there was a considerable improvement in the affective, cognitive and schizophrenic symptoms of a sample group, as opposed to the placebo group (Krzystanek et al., 2018). Telepsychiatry is also progressively used for opioid abuse patients in Northern Ontario, Canada (LaBelle et al, 2018). This implies that mobile telemedicine is a great tool for providing psychiatric care to people living in distant or remote areas.

A small $32.1 \%$ of respondents claimed that they would share information about smoking and alcohol through mobile applications. Interestingly, this result is quite similar to that of the European Commission for Bulgaria, which displays a $28 \%$ frequency of smoking.

The conclusions gathered with reference to body weight, nutrition and calorie intake are acutely befitting owing to the fact that according to WHO data, the Bulgarian nation has a high incidence of obesity. In conformity with the Pew Research Center, applications for physical activity, diet and body weight are the most popular smartphone health applications, with 38\% monitoring their physical activity, $31 \%$ diet and 12\% - body weight (Fox \& Duggan, 2012). In general, 27\% of adults in the United States use some form of health tracking technology (Fox \& Duggan, 2013).

The results show that the greatest proportion of the respondents was worried about difficulties in using mobile applications, as well as misinterpretation of information - 57\% and 58\% respectively. Moreover, a point that needs to be focused upon is that $42 \%$ of the respondents have concerns about the confidentiality of their personal health information and the possibility of it falling into the wrong hands. These fears are shared by other people in different parts of the world as well. For example, a South Korean study on real-time electrocardiographic monitoring found that the biggest hindrance in the use of these technologies was the concern about the safety and privacy of personal information (Lee et al, 2018). Another study examining the availability of a tele monitoring system for diabetes among doctors and patients in Austria found that major concerns were related to the protection of information and the loss of personal communication (Muigg et al, 2018).

The outcome of our study confirms and establishes the benefits of mobile health applications for patients, professionals and health organizations.

\section{Conclusion}

The study's conclusion is that a significant number of respondents were already using or were inclined to use smartphones and various applications, including health ones. We expect that the results of this study will provide evidence of the necessity for mobile health applications to be developed in Bulgaria. 
Additionally, the results would expand the usage of mobile health technologies, which would improve the quality of healthcare services provided to patients in Bulgaria. Health record events in Bulgaria will be a major plus point for the Bulgarian healthcare system.

In the nutshell, this study is a report on the general awareness and inclination to use mobile health applications among the Bulgarian population. Broadly speaking, the results show positive attitudes, while some socio-demographic factors still could obstruct the widespread adoption of mHealth technologies. Despite the fact that they acknowledge the advantages and efficiency of these technologies, such findings propose that users are quite reluctant and conservative about the security and privacy of their personal health data. Mobile health applications considerably eliminate informational inconsistencies, thus making consumers more dedicated towards their own health. All this calls for the necessary measures in order to enhance the awareness of people on the merits of health service provisions as provided through mHealth applications. The advancement of mHealth applications for patients with different diseases is a prerequisite for fresh opportunities in the monitoring and treatment of different socially significant diseases. Moreover, these could also offer resolution adoption, preventive measures and health promotion. Further investigations, evidence of success and conducive consolidation of patient-generated data into medical practice is imperative to build the reliability of people in the usage of mHealth applications to achieve public health goals in Bulgaria.

\section{Acknowledgement}

This research was supported by National Scientific Program "Electronic Health in Bulgaria" (eHealth) D-01-200/16.11.2018.

\section{References}

Anderson, R.M., Heesterbeek, H., Klinkenberg, D., Hollingsworth, T.D. (2020). How will country-based mitigation measures influence the course of the COVID-19 epidemic? Lancet. 395(10228): 931-934. doi: 10.1016/S0140-6736(20)30567-5

Asangansi, I., Braa, K. (2010). Safran, C., Reti, S., Marin, H.F. (eds.). The emergence of mobile-supported national health information systems in developing countries. MEDINFO 2010. Studies in health technology and informatics. 160. IOS Press. pp. 540-544.

Atallah, N., Khalifa, M., El Metwally, A., Househ, M. (2018). The Prevalence and usage of mobile health applications among mental health patients in Saudi Arabia. Computer Methods and Programs in Biomedicine. 156:163-168.

Chhabra, H.S., Sharma, S., Verma, S. (2018). Smartphone app in self-management of chronic low back pain: a randomized controlled trial. Eur Spine J. 27(11): 2862-2874. doi:10.1007/s00586-018-5788-5

Choi, P.J., Oskouian, R.J., Tubbs, R.S. (2018). Telesurgery: Past, Present, and Future. Cureus, 10(5), e2716.

https://doi.org/10.7759/cureus.2716

Currie, W.L. Seddon, J. (2014). mHealth in 28 EU Member States. TEMPEST mHealth. Retrieved from 11 August 2020:

https://vitaltransform.wpengine.com/wp-content/uploads/2014/11/TEMPEST-mobile.pdf

Debong, F., Mayer, H., Kober, J. (2019). Real-World Assessments of mySugr Mobile Health App. Diabetes Technology \& Therapeutics. 2019;21(S2): S235-S240. doi:10.1089/dia.2019.0019

Ericsson, E. (2015). Mobility Report: On the Pulse of the Networked Society. Retrieved from 31 May 2020: $\mathrm{https}: / /$ www.ericsson.com/assets/local/mobility-report/documents/2015/ericsson-mobility-report-june-2015.pdf.

Eurostat. (2020, 20 March). 53\% of EU citizens sought health information online. Eurostat. European Commission. Retrieved from 11 August 2020: https://ec.europa.eu/eurostat/en/web/products-eurostat-news/-/DDN-20200327-1

Fox, S., Duggan, M. (2012). Mobile Health 2012. Pew Internet. Pew Internet \& American Life Project. Pew Research Center. California Healthcare Foundation. Washington, D.C. Retrieved from 31 May 2020:

http://pewinternet.org/Reports/2012/Mobile-Health.aspx.

Fox, S., Duggan, M. (2013). Tracking for Health. Pew Internet. Pew Internet \& American Life Project. Pew Research Center. California Healthcare Foundation. Washington, D.C. Nov 8, 2012. Retrieved from 31 May 2020:

http://pewinternet.org/Reports/2013/Tracking-for-Health.aspx

Jeon, E.M., Seo, H.J. (2016). Acceptability of Service Targets for ICT-Based Healthcare. Healthcare Informatics Research. 22(4):333-341.

Hampton, T. (2012). Recent Advances in Mobile Technology Benefit Global Health, Research, and Care. JAMA;307(19): 2013-2014. doi: 10.1001/jama.2012.4465

Kemp, S. (2019). Digital 2019: Global Internet Use Accelerates. Retrieved from 31 May 2020: https://wearesocial.com/blog/2019/01/digital-2019-global-internet-use-accelerates.

Krzystanek, M., Borkowski, M., Krysta, K. (2018). Psychiatry as a leader of contemporary telemedicine in Poland. Reumatologia. 56(2):65-66.

LaBelle, B., Franklyn, A.M., Pkh Nguyen, V., Anderson, K.E., Eibl, J.K., Marsh, D.C. (2018). Characterizing the Use of Telepsychiatry for Patients with Opioid Use Disorder and Co-occurring Mental Health Disorders in Ontario, Canada. 
International Journal of Telemedicine and Applications; 2018:7937610.

https://www.ncbi.nlm.nih.gov/pmc/articles/PMC5828243/

Lee, S.J., Jung, T.Y., Lee, T.R., Han, J.H. (2018). Accepting telemedicine in a circulatory medicine ward in major hospitals in South Korea: patients' and health professionals' perception of real-time electrocardiogram monitoring. BMC Health Services Res. 2018;18(1):293.

Marcolino, S.M., Oliveira, Q.J.A., D'Agostino, M., Ribeiro, L.A., Alkmim, M.M.B., Novillo-Ortiz, D. (2018). The Impact of mHealth Interventions: Systematic Review of Systematic Reviews. JMIR mHealth uHealth. 6(1):e23.

McKay, F.H., Cheng, C., Wright, A., Shill, J., Stephens, H., Uccellini, M. (2018). Evaluating mobile phone applications for health behaviour change: A systematic review. Journal of Telemedicine and Telecare. 24(1): 22-30. doi: $10.1177 / 1357633 X 16673538$

Mohapatra, D., Mohapatra, M., Chittoria, R., Friji, M., Kumar, S. (2015). The scope of mobile devices in health care and medical education. Int J Adv Med Health Res. 2(1):3-8.

Muigg, D., Kastner, P. Modre-Osprian, R., Haluza, D., Duftschmid, G. (2018). Is Austria Ready for Telemonitoring? A Readiness Assessment Among Doctors and Patients in the Field of Diabetes. Studies in Health Technology and Informatics. 248:322-329. https://www.ncbi.nlm.nih.gov/pubmed/29726454

Oliver, N., Lepri, B.; Sterly, H., Lambiotte, R., Delataille, S., De Nadai, M., Letouzé, E., Salah, A. A., Benjamins, R., Cattuto, C., et al. (2020) Mobile phone data for informing public health actions across the COVID-19 pandemic life cycle. Sci. Adv. 2020. doi: 10.1126/sciadv.abc0764.

Sindi, S., Calov, E., Fokkens, J., et al. (2015). The CAIDE Dementia Risk Score App: The development of an evidence-based mobile application to predict the risk of dementia. Alzheimers Dement (Amst). 1(3): 328-33.

Tchero, H., Kangambega, P., Briatte, C., Brunet-Houdard, S., Retali, G.R., Rusch, E. (2019). Clinical Effectiveness of Telemedicine in Diabetes Mellitus: A Meta-Analysis of 42 Randomized Controlled Trials. Telemed J E Health. 25(7): 569583. doi: 10.1089/tmj.2018.0128

Vinarova, Z. (2015). Informacionna paradigm $v$ zdraveopazvaneto [Information paradigm in healthcare]. Izd.: Zahari Stoyanov, Sofia

Wallace, L.S., Dhingra, L.K. (2014). A systematic review of smartphone applications for chronic pain available for download in the United States. J Opioid Manag. 10(1): 63-68. doi: 10.5055/jom.2014.0193

Wattanapisit, A., Teo, C.H., Wattanapisit, S. et al. (2020). Can mobile health apps replace GPs? A scoping review of comparisons between mobile apps and GP tasks. BMC Medical Informatics and Decision Making (2020), 20:5. https://doi.org/10.1186/s12911-019-1016-4

WHO Global Observatory for eHealth. (2011). mHealth: new horizons for health through mobile technologies: second global survey on eHealth. Geneva. World Health Organization. Retrieved from 31 May 2020: https://apps.who.int/iris/handle/10665/44607

World Bank Group. Mobile cellular subscriptions. Retrieved from 31 May 2020: https://data.worldbank.org/indicator/IT.CEL.SETS

World Health Organization. (2016). 139 th session (27 May 2016) Provisional agenda item 6.6 mHealth: use of mobile wireless technologies for public health. Retrieved from 31 May 2020: http://apps.who.int/gb/ebwha/pdf_files/EB139/B139_8-en.pdf?ua=1.

World Health Organization. (2018). Seventy-first World Health Assembly. Agenda item 12.4. Digital health. WHA 71.7. 26 May 2018. Retrieved from 31 May 2020: http://apps.who.int/gb/ebwha/pdf_files/WHA71/A71_R7-en.pdf?ua=1 